\title{
The Use of Parameter-Free Correlation Functions in Green's Function Monte Carlo Simulations of Small Electronic Systems
}

\author{
C. Mecke and F. F. Seelig \\ Institut für Physikalische und Theoretische Chemie, Eberhard-Karls-Universität Tübingen, \\ D-72076 Tübingen, Germany
}

Z. Naturforsch. 52 a, 793-802 (1997); received October 11, 1997

Using an old formulation for correlation functions with correct cusp-behaviour, the Schrödinger equation transforms to a new differential equation which provides a very simple expression for the local electronic energy with limited range. This, together with the simplicity of the formulation promises a high performance in Green's function Monte Carlo (GFMC) simulations of small electronic systems. The behaviour of the local energy is studied on a few simple examples because the variance of this function determines the quality of the results in the GFMC methods. Calculations for one- and two-electron systems are presented and compared with results from well-known functions. The form of the function is then extended to systems with more than two electrons. Results for the $\mathrm{Be}$ atom are given and the extension to larger electronic systems is discussed.

Key words: Correlation Functions, Green's Function Monte Carlo, Local Energy, Wave Functions with Correct Cusp-behaviour, Jastrow Factors

\section{Introduction}

Greens's function quantum Monte Carlo methods have been an intensively examined branch of quantum mechanics in the last years. The method has now become well established and standard programs for calculations of smaller electronic systems are available [1]. Progress still has to be done in extending the method on larger systems. Questions of the node problem in the case of fermion systems have to be solved, and the convergence behaviour of such calculations is to be improved. The latter can be achieved by developing more accurate guiding functions with a very small variance of their corresponding local energies. An important point to keep in mind is of course that better guiding functions are of an increasing complexity, which causes more computational effort. Therefore care must be taken that the advantages of better guiding functions are not overcompensated by the disadvantage of increasing CPU-time needed for evaluating the functions millions of times. A simple formulation like the one we suggest could, in spite of being not so sophisticated as other ones, be superior in the computational practice. In addition to that,

Reprint requests to Prof. F. F. Seelig, Fax: +497071 296910. the question of the minimum requirements of a formulation without parameters, which would have been determined first in another calculation, remains an interesting point in ab-initio quantum chemistry.

The starting point of the following inspections is the time-independent Schrödinger equation

$$
\mathcal{H} \Psi_{p}=E_{p} \Psi_{p}
$$

with the Hamiltonian $\mathcal{H}$, the wave function $\Psi_{p}$ which for simplicity here is taken to be real, and the corresponding energy $E_{p}$ of the $p^{\text {th }}$ quantum state. The quantum mechanical, non-relativistic, electronic Hamiltonian for a molecule of $N$ electrons with the coordinates $\vec{r}_{i}=\left(x_{i}, y_{i}, z_{i}\right)(i=1,2, \ldots, N)$ in the field of $M$ nuclei with the coordinates $\vec{r}_{K}=$ $\left(x_{k}, y_{K}, z_{K}\right)$ and the charge numbers $Z_{K}(K=$ $1,2, \ldots, M)$ in a Cartesian coordinate system within the Born-Oppenheimer approximation using atomic units is

$$
\mathcal{H}_{\mathrm{el}}=-\frac{1}{2} \sum_{i=1}^{N} \nabla_{i}^{2}-\sum_{i=1}^{N} \sum_{K=1}^{M} \frac{Z_{K}}{r_{i K}}+\sum_{i=1}^{N-1} \sum_{j=i+1}^{N} \frac{1}{r_{i j}}
$$

( $r_{i K}=\left|\vec{r}_{i}-\vec{r}_{K}\right|$ is the distance between electron $i$ and nucleus $K$ and $r_{i j}=\left|\vec{r}_{i}-\vec{r}_{j}\right|$ is the inter-electronic distance). 
With the new quantity

$$
\rho=\sum_{i=1}^{N} \sum_{K=1}^{M} Z_{K} r_{i K}-\frac{1}{2} \sum_{i=1}^{N-1} \sum_{j=i+1}^{N} r_{i j}
$$

introduced in [2], [3] the ground state wave function $\Psi$ (where in the following the ground state index $p=0$ for simplicity is omitted) can be written as

$$
\Psi=e^{-\rho} F
$$

Inserting this formulation in the Schrödinger equation produces a new differential equation for the function $F$ :

$$
-\frac{1}{2} \sum_{i=1}^{N}\left[\nabla_{i}^{2}-2\left(\nabla_{i} \rho \cdot \nabla_{i}\right)+\left(\nabla_{i} \rho\right)^{2}\right] F=E F .
$$

The expression for the local energy of a trial function $\Phi=e^{-\rho} F$,

$$
\varepsilon\left(\vec{r}_{1}, \vec{r}_{2}, \ldots, \vec{r}_{N}\right)=\frac{\mathcal{H} \Phi}{\Phi}
$$

therefore can be written as

$$
\varepsilon=-\frac{1}{2} \frac{\sum_{i=1}^{N}\left[\nabla_{i}^{2}-2 \nabla_{i} \rho \cdot \nabla_{i}\right] F}{F}+V^{\prime},
$$

introducing a new pseudo-potential

$$
V^{\prime}=-\frac{1}{2} \sum_{i=1}^{N}\left(\nabla_{i} \rho\right)^{2}
$$

It turns out that our formulation of the wave function produces at least three attractive properties [4]. These are the fulfilment of the cusp-conditions [5], the exact compensation of the true coulomb potential and the generation of a new pseudo-potential which is bounded from above and from beyond. The expression for the local energy, due to the special choice for the ground state wave function with correct cuspbehaviour, therefore has very attractive properties for the use in some numerical quantum mechanical methods, as will be shown in the next sections.

\section{Method}

Originally the method of the Green's function Monte Carlo calculations was introduced by Kalos [6], Anderson [7-9] and others [10]. Here we only have to point to a few basic key ideas. Details of the implementation can be found in these papers and in the textbook of Hammond, Lester, and Reynolds [11], where the method we use is often referred as "Bessel function Monte Carlo (BFMC)".

Assuming that the potential in the Schrödinger equation had an upper bound $V_{\max }>0$, the expression $\left(V(\vec{R})-V_{\max }\right)$, would always be negative (or zero) (in the following capital letters $\vec{R}=$ $\left(x_{1}, y_{1}, z_{1}, \ldots, x_{N}, y_{N}, z_{N}\right)$ are used for positions of the $3 N$-dimensional, electronic space, while small letters $\left(\vec{r}_{i}\right)$ denote the coordinates of a single electron). Therefore, to apply this method the electron-electron interaction of the true coulomb potential $V_{\mathrm{C}}$

$$
V_{\mathrm{C}}=-\sum_{i=1}^{N} \sum_{K=1}^{M} \frac{Z_{K}}{r_{i K}}+\sum_{i=1}^{N-1} \sum_{j=i+1}^{N} \frac{1}{r_{i j}}
$$

has to be cut off at $V_{\mathrm{C}}=V_{\max }$ (this is equivalent to an introduction of a minimal electron-electron distance $r_{0}$ ). Of course this assumption introduces a small error which depends on the choice of $V_{\max }$ and can be related to the time step error in Diffusion Monte Carlo methods (DMC). Additional considerations are necessary to get the best value for $V_{\max }$. For one-electron systems this method provides an absolutely accurate way for computational calculations. The Schrödinger equation with this shift parameter $V_{\max }$ now reads

$$
\left[-\frac{1}{k^{2}} \nabla^{2}+1\right] \Psi(\vec{R})=\left[\frac{V(\vec{R})-V_{\max }}{E_{0}-V_{\max }}\right] \Psi(\vec{R}),
$$

where we have introduced the new parameter $k^{2}=$ $-2\left(E_{0}-V_{\max }\right)$ for convenience, and $\nabla^{2}$ is based on the coordinates $\vec{R}$ of all the electrons. The expression in brackets on the left-hand side of (2) is an Helmholtz operator. The Green's function of this operator is wellknown:

$$
G_{\text {Bess }}\left(\vec{R}^{\prime}, \vec{R}\right)=(2 \pi)^{-(\nu+1)} R^{-\nu} K_{\nu}(R)
$$

( $\left.R=k\left|\vec{R}-\vec{R}^{\prime}\right|, \nu=3 N / 2-1\right) . K_{\nu}$ is the modified (spherical) Bessel function (if $\nu$ is integer, $K_{\nu}$ is 
of second, in the other cases of third kind [12]). Introducing a guiding function $\Phi$ in order to perform a Metropolis acceptance step in the simulation, the final integral equation which has to be solved iteratively by Monte Carlo methods is

$$
\begin{aligned}
& f\left(\vec{R}^{\prime}\right)=\Phi\left(\vec{R}^{\prime}\right) \Psi_{0}\left(\vec{R}^{\prime}\right) \\
& =\int_{-\infty}^{\infty} \frac{\Phi\left(\vec{R}^{\prime}\right)}{\Phi(\vec{R})} G_{\text {Bess }}\left(\vec{R}^{\prime}, \vec{R}\right)\left[\frac{V(\vec{R})-V_{\max }}{E_{0}-V_{\max }}\right] f(\vec{R}) \mathrm{d} \vec{R} .
\end{aligned}
$$

The electronic ground state energy can be calculated by summation of the local energies $\varepsilon_{i}$ of the set of $3 N$-dimensional points $\left\{\vec{R}_{i}\right\}$ ("walkers") in a random walk algorithm, which after the simulation are distributed like the mixed function $f$ :

$$
E_{0}=\langle\varepsilon\rangle_{f}=\lim _{M \rightarrow \infty} \frac{1}{M} \sum_{i=1}^{M} \varepsilon\left(\vec{R}_{i}\right) .
$$

Care must be taken to avoid statistical correlation.

In the case of systems with more than two electrons the wave function of fermion systems $\Psi^{F}$ has to be antisymmetric under exchange of the space- and spincoordinates of two particles:

$$
\begin{aligned}
& \Psi^{F}\left(\ldots,\left\{\vec{r}_{i}, \omega_{i}\right\}, \ldots,\left\{\vec{r}_{j}, \omega_{j}\right\}, \ldots\right) \\
& =-\Psi^{F}\left(\ldots,\left\{\vec{r}_{j}, \omega_{j}\right\}, \ldots,\left\{\vec{r}_{i}, \omega_{i}\right\}, \ldots\right) .
\end{aligned}
$$

This constraint is usually achieved by a determinant form of the guiding function. Using a determinant specifies the nodes of the function. Because of the lack of knowledge of the general shape of the nodes [13], [14], an additional error is introduced when the common fixed-node approximation [8] of the BFMC method is implemented.

\section{Guiding Functions}

The most common formulation for guiding functions in GFMC methods is of the correlated molecular orbital (CMO) type:

$$
\Phi_{\mathrm{CMO}}=\Phi_{\mathrm{C}} \Phi_{\mathrm{D}}
$$

where $\Phi_{\mathrm{C}}$ denotes a correlation factor and $\Phi_{\mathrm{D}}$ a determinant form. For $\Phi_{\mathrm{C}}$ very often Jastrow factors are used:

$$
\Phi_{\mathrm{C}}=e^{-\rho^{J}},
$$

where

$$
\rho^{J}=\sum_{i=1}^{N} \rho_{i}^{J}+\rho_{\text {corr }}^{J}
$$

with the simplest form of a Padé-approximation

$$
\rho_{i}^{J}=\sum_{K=1}^{M} \frac{\lambda_{K} r_{i K}}{1+\nu_{K} r_{i K}}
$$

and

$$
\rho_{\text {corr }}^{J}=\sum_{i=1}^{N-1} \sum_{j=i+1}^{N} \frac{a r_{i j}}{1+b r_{i j}},
$$

where the parameter $a$ has to be chosen as $a=-1 / 2$ in order to satisfy the electron-electron cusp condition. The parameters $\lambda_{K}$ have to be determined to fulfil the electron-nucleus cusp conditions. The $\nu_{K}$ and $b$ can be used to minimize either the expectation value of the guiding function or the variance of its local energy function. Although there are a lot of values for these parameters tabulated, the work for this optimisation has to be taken into account for a fair comparison of the computational effort for different guiding functions. Obviously our formulation is the simplest form of a Jastrow factor with correct cusp-behaviour without any parameters.

The pseudo-potential $V^{\prime}$ in the expression for the local energy of our formulation has some remarkable properties. As we can see from (1), it becomes identical with the local energy if we use $F=1$ as an expression for the unknown function $F$. For systems up to two electrons with singlet ground state this becomes a possible and attractive choice. Under closer inspection of the expression for $V^{\prime}$,

$$
\begin{aligned}
& V^{\prime}=-\frac{1}{2} \sum_{i=1}^{N}\left(\nabla_{i} \rho\right)^{2} \\
&=-\frac{1}{2}\left[\sum_{i=1}^{N} \sum_{K=1}^{M} \sum_{\substack{L=1 \\
L}}^{M} Z_{K} Z_{L}\left(\boldsymbol{r}_{i K} \boldsymbol{r}_{i L}\right)\right. \\
&-\sum_{i=1}^{N} \sum_{\substack{j=1 \\
j \neq i}}^{N} \sum_{K=1}^{M} Z_{K}\left(\boldsymbol{r}_{i K} \boldsymbol{r}_{i j}\right) \\
&\left.+\frac{1}{4} \sum_{i=1}^{N} \sum_{\substack{j=1 \\
j \neq i \\
j \neq i}}^{N} \sum_{\substack{l=1 \\
l \neq i}}^{N}\left(\boldsymbol{r}_{i j} \boldsymbol{r}_{i l}\right)\right],
\end{aligned}
$$




$$
\begin{array}{r}
V^{\prime}=-\frac{1}{2}\left[N\left[\sum_{K=1}^{M} Z_{K}^{2}+\frac{1}{4}(N-1)\right]\right. \\
+\sum_{i=1}^{N}\left[\sum_{K=1}^{M} \sum_{\substack{L=1 \\
L \neq K}}^{M} Z_{K} Z_{L}\left(\boldsymbol{r}_{i K} \boldsymbol{r}_{i L}\right)\right. \\
-\sum_{\substack{j=1 \\
j \neq i}}^{N} \sum_{K=1}^{M} Z_{K}\left(\boldsymbol{r}_{i K} \boldsymbol{r}_{i j}\right) \\
\left.\left.+\frac{1}{4} \sum_{\substack{j=1 \\
j \neq i}}^{N} \sum_{\substack{l=1 \\
l \neq j \\
l \neq j}}^{N}\left(\boldsymbol{r}_{i j} \boldsymbol{r}_{i l}\right)\right]\right]
\end{array}
$$

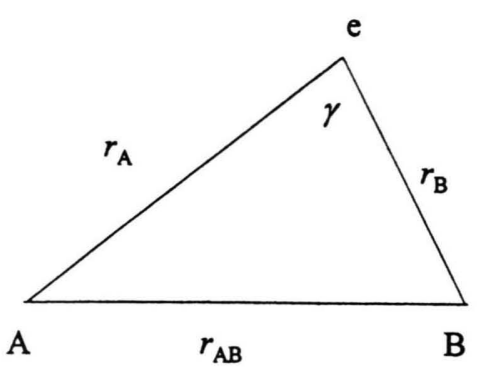

where

$$
\left(\boldsymbol{r}_{i j} \boldsymbol{r}_{i l}\right)=\nabla_{i} r_{i j} \cdot \nabla_{i} r_{i l}=\frac{r_{i j}^{2}+r_{i l}^{2}-r_{j l}^{2}}{2 r_{i j} r_{i l}}=\cos \vartheta_{j i l}
$$

$\left(\vartheta_{j i l}\right.$ is the angle between the two vectors $\boldsymbol{r}_{i j}$ and $\boldsymbol{r}_{i l},\left(\boldsymbol{r}_{i K} \boldsymbol{r}_{i L}\right)$ and $\left(\boldsymbol{r}_{i K} \boldsymbol{r}_{i j}\right)$ are defined in an analogous way), it turns out that it is very easy to determine an upper and lower bound for the local energy. The upper bound could be set to zero and for the lower bound $S$,

$$
\begin{aligned}
S=-\frac{1}{2} N\left[\sum_{K=1}^{M} \sum_{L=1}^{M} Z_{K} Z_{L}+(N-1)\right. & \sum_{K=1}^{M} Z_{K} \\
& \left.+\frac{1}{4}(N-1)^{2}\right]
\end{aligned}
$$

is a possible choice. In most examples an even better bound can be determined. This becomes important because in QMC methods the variance of the local energy determines significantly the quality of the result.

\section{Examples}

\section{Systems with up to Two Electrons}

In order to get a better impression of our guiding functions we studied a few simple examples, where we are able to visualise the properties of the local energy. For the hydrogen molecular ion (Fig. 1) the exact solution of the corresponding Schrödinger equation is possible [15] and the $\sum^{+}$-orbital is shown in Figure 2. Thus the exact function $F$ (Fig. 3) can easily be gained by multiplying the exact solution with $e^{\rho}$ (note the plateau between the nuclei in Figure 4). The pseudo-potential for the hydrogen molecular ion $\left(Z_{A}=Z_{B}=Z=1\right)$ is simply

Fig. 1. Coordinates of the hydrogen molecular ion.

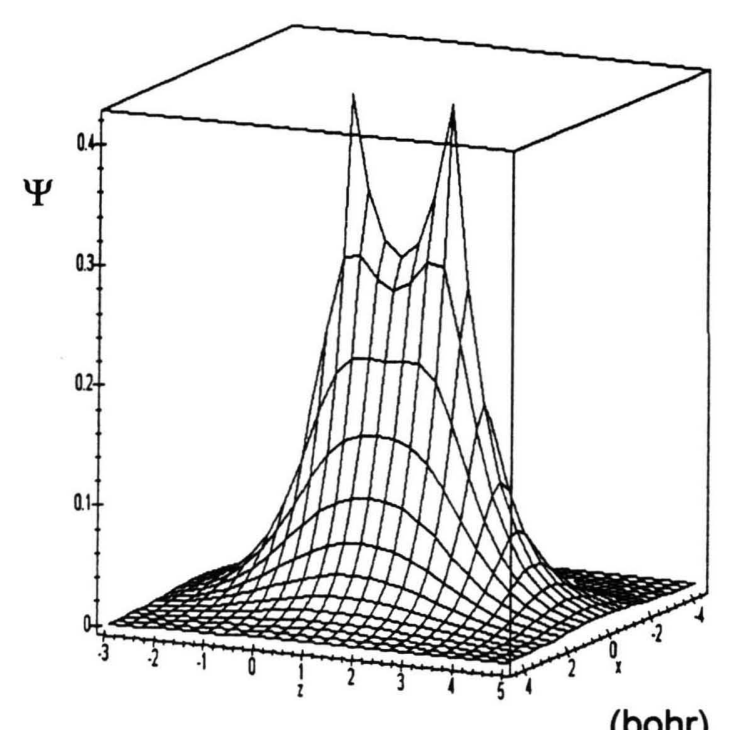

Fig. 2. $\sum^{+}$-orbital of the hydrogen molecular ion (3D) from the original work [15] $(y=0)$ in atomic units.

$$
V^{\prime}=-\frac{1}{2}\left(Z_{A}^{2}+Z_{B}^{2}+2 Z_{A} Z_{B}(\underbrace{\boldsymbol{r}_{A} \boldsymbol{r}_{B}}_{\cos (\gamma)})\right)
$$

and remains inside the interval $\left[-1 / 2\left(Z_{A}+Z_{B}\right)^{2}\right.$, $\left.-1 / 2\left(Z_{A}-Z_{B}\right)^{2}\right]$. The difference between the upper and the lower bound therefore is $\Delta V^{\prime}=2 Z_{A} Z_{B}$. Figures 5 and 6 show the pseudo-potential along the axis of the two nuclei $(x=y=0)$.

We compared this function with a second guiding function $\Phi_{2}$, which was a simple LCAO-function

$$
\Phi_{2}=N_{2}\left(\varphi\left(r_{A}\right)+\varphi\left(r_{B}\right)\right)
$$

where $\varphi(r)=e^{-\alpha r}$ (for $\alpha$, the optimum exponent for the internuclear distance of 2.0 a.u., is used: 


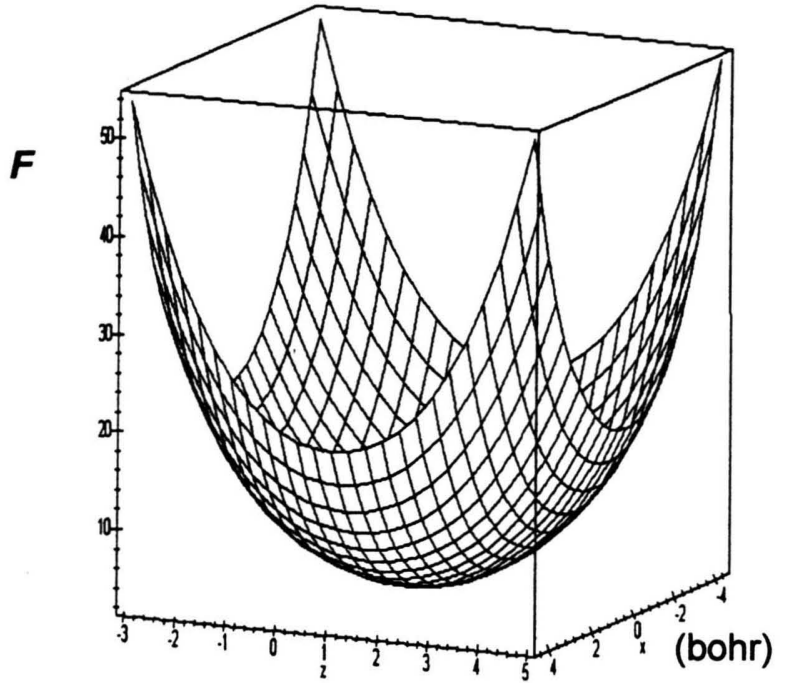

Fig. 3. Function $F$ of the hydrogen molecular ion (3D), gained with the original work [15] $(y=0)$ in atomic units.

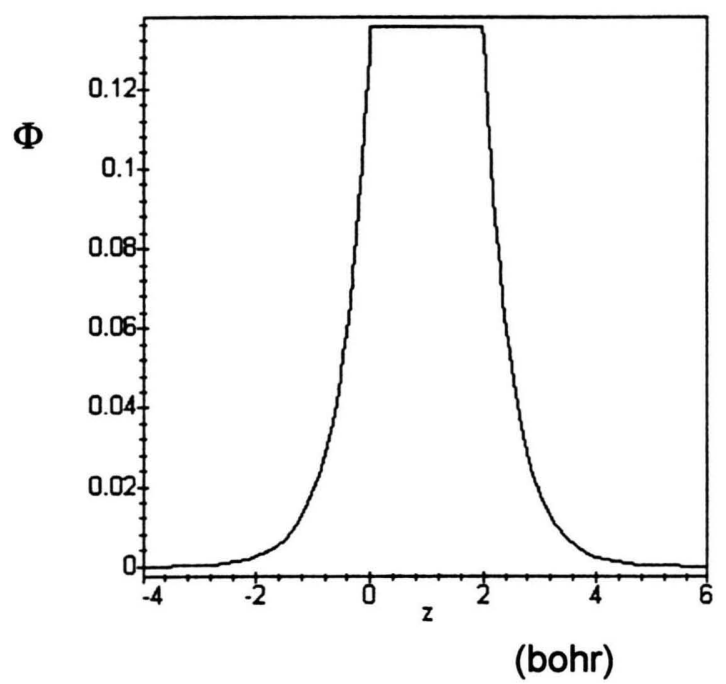

Fig. 4. $\Phi=e^{-\rho}$ for the hydrogen molecular ion, section along the nuclear axis $(x=y=0)$ in atomic units.

$\alpha=1.25)$. Because of the wrong cusp-behaviour the local energy of this function is of course not bounded:

$$
\begin{aligned}
\varepsilon= & \frac{N_{2}}{\Phi_{2}}\left(\left(\frac{\alpha}{r_{A}}-\frac{\alpha^{2}}{2}\right) \varphi\left(r_{A}\right)+\left(\frac{\alpha}{r_{B}}-\frac{\alpha^{2}}{2}\right) \varphi\left(r_{B}\right)\right) \\
& -\frac{Z}{r_{A}}-\frac{Z}{r_{B}} .
\end{aligned}
$$

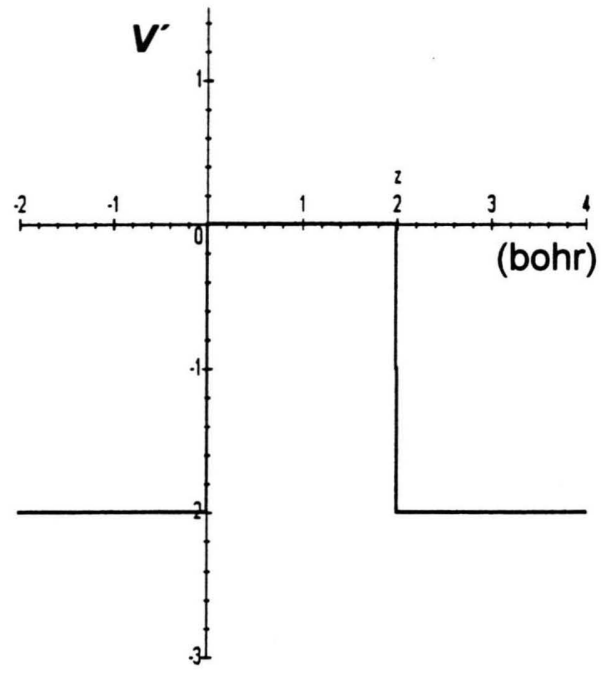

Fig. 5. Pseudo-potential of the hydrogen molecular ion, section along the nuclear axis $(x=y=0)$ in atomic units.

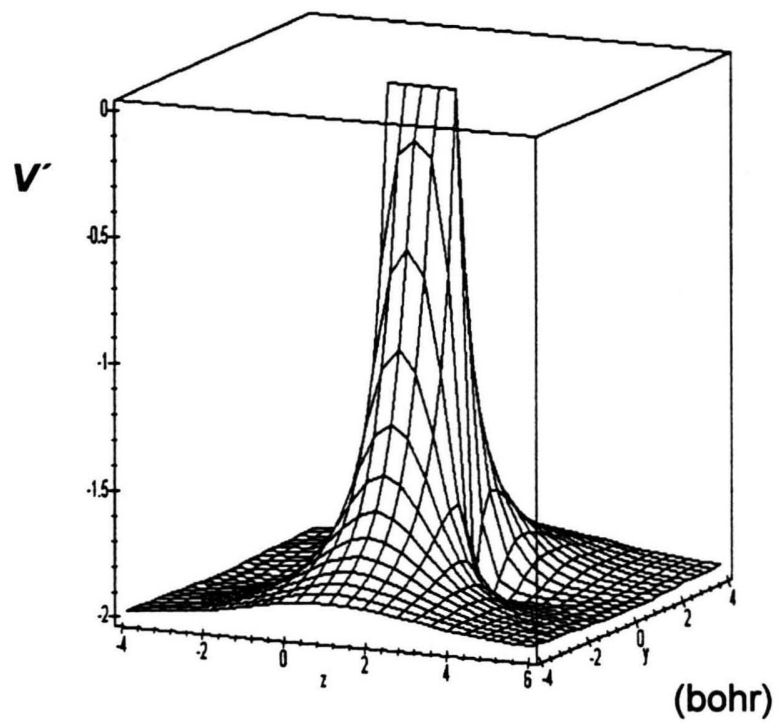

Fig. 6. 3-dimensional graph of the pseudo-potential of the hydrogen molecular ion $(x=0)$ in atomic units.

For the helium atom (Fig. 7) the pseudo-potential within our formulation is

$V^{\prime}=\frac{Z}{2}(\underbrace{\frac{r_{1}^{2}+r_{12}^{2}-r_{2}^{2}}{2 r_{1} r_{12}}}_{\cos (\alpha)}+\underbrace{\frac{r_{2}^{2}+r_{12}^{2}-r_{1}^{2}}{2 r_{2} r_{12}}}_{\cos (\beta)})-Z^{2}-\frac{1}{4}$ 


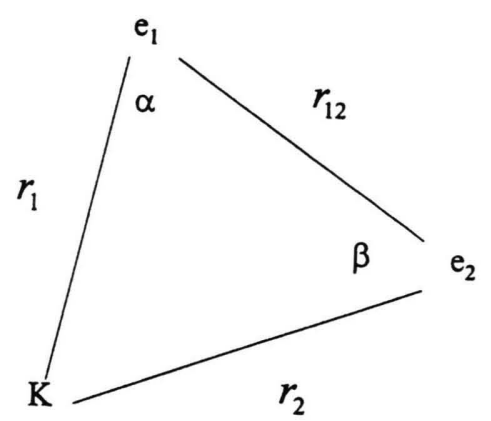

Fig. 7. Coordinates of the helium atom.

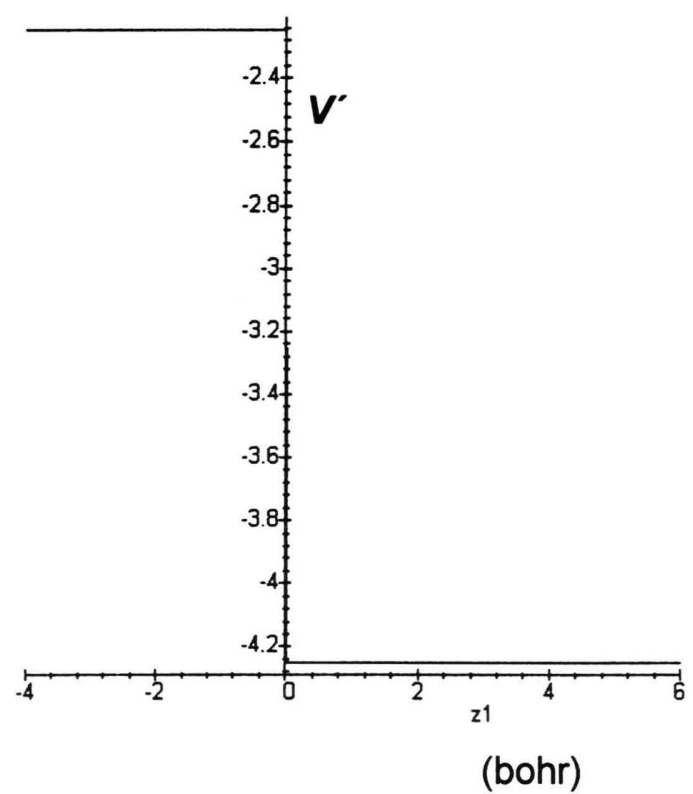

Fig. 8. Pseudo-potential $V^{\prime}$ of the He atom for electron one along the $z_{1}$-axis (electron 2: $x_{2}=0, y_{2}=0, z_{2}=2$ ) in atomic units.

Because the sum $(\cos (\alpha)+\cos (\beta))$ is bounded in the interval $[0,2]$, the pseudo-potential $V^{\prime}$ is between $-\left(Z^{2}+1 / 4\right)$ and $-\left(Z^{2}-Z+1 / 4\right)$. The difference between the upper and lower bound therefore is simply $\Delta V^{\prime}=Z$. Figures 8 and 9 show the pseudo-potential holding the position of electron 2 fixed at $\left(x_{2}=0\right.$, $y_{2}=0, z_{2}=2$ ). In Fig. 8 the first electron moves along the $z_{1}$-axis, in Fig. 9 along the $y_{1}$-axis. Note that in Fig. 8 the position of electron 2 can not be seen. A three dimensional representation is given in Figure 10.

For the purpose of comparison we used a "singlezeta" Hartree-Fock function

$$
\Phi_{\mathrm{HF}}=N \phi(1) \phi(2)
$$

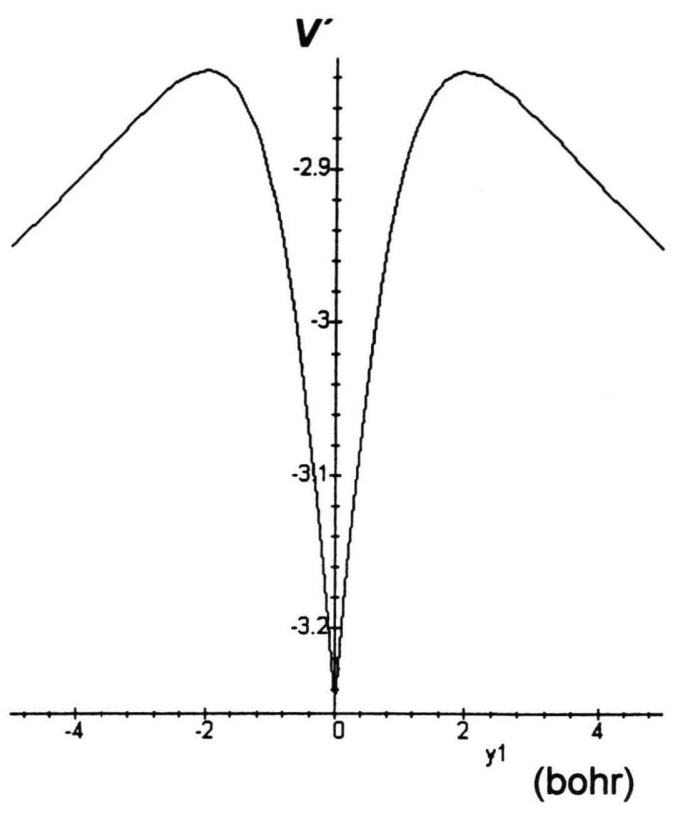

Fig. 9. Pseudo-potential $V^{\prime}$ of the He atom for electron one along the $y_{1}$-axis (electron 2: $x_{2}=0, y_{2}=0, z_{2}=2$ ) in atomic units.

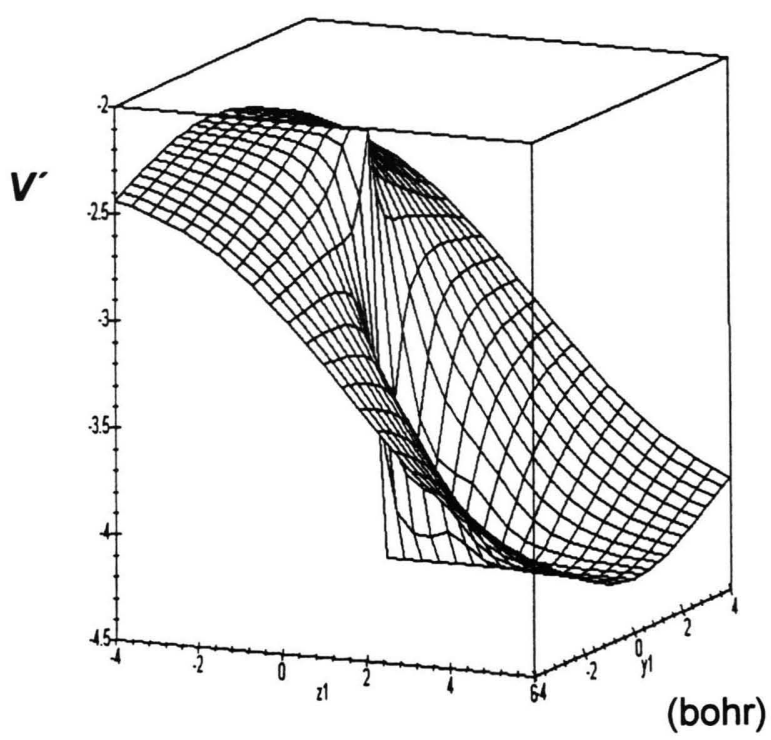

Fig. 10. Pseudo-potential $V^{\prime}$ of the He atom for electron one (electron 2: $x_{1}=0, x_{2}=0, y_{2}=0, z_{2}=2$ ) in atomic units.

where $\phi(r)=e^{-\zeta r}(\zeta=11 / 16)$. Due to the wrong cusp-behaviour the expression for the local energy $\varepsilon_{\mathrm{HF}}$, 


$$
\varepsilon_{\mathrm{HF}}=-\zeta^{2}+(\zeta-Z)\left(\frac{1}{r_{1}}+\frac{1}{r_{2}}\right)+\frac{1}{r_{12}}
$$

again is not bounded.

\section{Systems with more than Two Electrons}

For electronic systems with more than 2 electrons the choice of $F=1$ is no longer possible. To guarantee the antisymmetry of the guiding function we use the common formulation

$$
F=A(1, \ldots, N / 2) B(N / 2+1, \ldots, N)
$$

where

$$
A(1, \ldots, N / 2)=\left|\begin{array}{llll}
\phi_{1}(1) & \phi_{2}(1) & \cdots & \phi_{N / 2}(1) \\
\phi_{1}(2) & & & \\
\vdots & & \ddots & \\
\phi_{1}(N / 2) & & &
\end{array}\right|
$$

( $\phi_{i}$ are molecular orbitals, $B$ is defined in an analogous way). Hereby the closed-shell case is assumed. Taking the $\mathrm{Be}$ atom as an example we use the sorbitals $(l=0)$ of the hydrogen atom and related ions with higher nuclear charge in order to get a simple and parameter free model for the two determinants $A$ and $B$ :

$$
R_{n}(r)=N_{n} L_{n}^{1}\left(\frac{2 Z}{n} r\right) e^{-\frac{Z r}{n}}
$$

(normalisation constants $N_{n}$ are omitted in the following, $L_{n}^{1}$ are the associated Laguerre polynomials of order 1 and degree $n$ ). For Be we use the 1s- and 2s-orbitals:

$$
\begin{aligned}
& \psi_{1 \mathrm{~s}}(r)=e^{-Z r} \\
& \psi_{2 \mathrm{~s}}(r)=\left(1-\frac{Z r}{2}\right) e^{-\frac{Z r}{2}}
\end{aligned}
$$

(In order to conserve the cusp-condition it is necessary to use the full nuclear charge $Z$ and not an effective nuclear charge $Z_{\text {eff }}$ according to the Slater rules for example). Extracting the $e^{-\rho}$-factor out of the s-orbitals we finally get for the guiding function

$$
\begin{aligned}
\Phi_{1} & =N_{1} e^{-\rho}\left|\begin{array}{ll}
1 & \phi_{2}(1) \\
1 & \phi_{2}(2)
\end{array}\right|\left|\begin{array}{ll}
1 & \phi_{2}(3) \\
1 & \phi_{2}(4)
\end{array}\right| \\
& =N_{1} e^{-\rho}\left(\phi_{2}(2)-\phi_{2}(1)\right)\left(\phi_{2}(4)-\phi_{2}(3)\right)
\end{aligned}
$$

and therefore for the "orbitals" in the original determinant form of (3)

$$
\begin{aligned}
& \phi_{1}(r)=1 \\
& \phi_{2}(r)=\left(1-\frac{Z r}{2}\right) e^{\frac{Z r}{2}} .
\end{aligned}
$$

Note that the functions $\phi_{1}$ and $\phi_{2}$ have no influence on the correct electron-nucleus cusp-behaviour of our formulation (this can be seen by the absence of the first power of $r$ in the corresponding Taylor series of $\phi_{1}$ and $\left.\phi_{2}\right)$.

Again a second guiding function is used, where we chose a single-zeta Hartree-Fock function

$$
\Phi_{\mathrm{HF}}=N_{2}|\underbrace{\begin{array}{ll}
\phi_{1}(1) & \phi_{2}(1) \\
\phi_{1}(2) & \phi_{2}(2)
\end{array}}_{A}| \underbrace{\begin{array}{ll}
\phi_{1}(3) & \phi_{2}(3) \\
\phi_{1}(4) & \phi_{2}(4)
\end{array}}_{B} \mid
$$

with the two molecular orbitals

$$
\begin{aligned}
& \phi_{1}=c_{11} \varphi_{1 \mathrm{~s}}+c_{21} \varphi_{2 \mathrm{~s}}, \\
& \phi_{2}=c_{12} \varphi_{1 \mathrm{~s}}+c_{22} \varphi_{2 \mathrm{~s}}
\end{aligned}
$$

$\left(c_{11}=1.0, c_{21}=0.7 ; c_{12}=0.7, c_{22}=1.0\right)$ and the (normalised) Slater basis functions $\left(\zeta_{1 \mathrm{~s}}=3.68478\right.$, $\left.\zeta_{2 \mathrm{~s}}=0.95603\right)$ :

$$
\begin{aligned}
& \varphi_{1 \mathrm{~s}}=\sqrt{\frac{\zeta_{1 \mathrm{~s}}^{3}}{\pi}} e^{-\zeta_{1 \mathrm{~s}} r}, \\
& \varphi_{2 \mathrm{~s}}=\sqrt{\frac{\zeta_{2 \mathrm{~s}}^{5}}{3 \pi}} r e^{-\zeta_{2 \mathrm{~s}} r} .
\end{aligned}
$$

The extension of this model on larger systems is possible, but numerical results show that the node structure of guiding functions gained by this simple formulation seems to get worse especially for the multicentre case of molecules. In addition to that, studies of this model for the systems $\mathrm{LiH}, \mathrm{Li}_{2}$ and $\mathrm{H}_{2} \mathrm{O}$ reveal that numerical problems in the convergence behaviour have to be expected.

\section{Results and Discussion}

Calculations for the Variational Monte Carlo (VMC) and the BFMC method were performed with a 1300-lines-FORTRAN program. VMC was used in order to calculate the expectation values where no 
analytic solution is available and to calculate the variances $\sigma^{2}$ of the local energy function. For each example we used a starting population of 10000 points ("walkers") of the $3 \mathrm{~N}$-dimensional electronic space, which was generated in a first run. Starting from this population, 10 independent calculations of $10^{5}$ Monte Carlo cycles were done with different starting values of the random number generator. The first 500 cycles were discarded before data was gathered. The numbers in brackets are two times the standard error of the last given decimal.

In a first step we did a few calculations on oneelectron systems in order to get a feeling for systematic errors for the more difficult examples later, and to have a reference for the accuracy which can be expected for the chosen computational effort (remember that for the one electron systems the BFMC method is exact). The simplest example is the hydrogen atom. Because our formulation in this special case provides the exact solution for the ground state, it is not possible to use it as a guiding function (the local energy would become the constant eigenvalue). Instead we used an optimised Gauss function and a second function with the same expectation value but correct cusp-behaviour $(\beta=1.848374 Z)$ :

$$
\Phi_{2}=N_{2}(1+(\beta-Z) r) e^{-\beta r} .
$$

The standard deviations were calculated for the BFMC and the Variation Monte Carlo method. As expected, the value of the standard deviation $\sigma$ for the Gauss function is approximately twice as high as for $\Phi_{2}$. According to the law of convergence for Monte Carlo methods,

$$
\sigma_{\bar{x}}^{2}=\frac{\sigma^{2}}{N}
$$

( $\sigma_{\bar{x}}^{2}$ is the variance of the mean and $N$ here denotes the number of sample points), the consequence of the higher standard deviation of the Gauss function can be directly seen in the error bar $\left( \pm 2 \sigma_{\bar{x}}\right)$ for the value of the energy. Note the difference in the error bars for the standard deviations themselves.

For the hydrogen molecular ion our formulation has the disadvantage of having an unphysical plateau between the two nuclei as already discussed (Figure 4). Therefore the variance of the local energy is worse than that of the LCAO-formulation in spite of the correct cusp-behaviour of our guiding function.
Table 1. Results of the one-electron systems (energies in

\begin{tabular}{|c|c|c|c|c|c|}
\hline & $\langle E\rangle_{\mathrm{VMC}}$ & $\langle E\rangle_{\mathrm{BFMC}}$ & $E_{\exp }$ & $\sigma_{\mathrm{VMC}}$ & $\sigma_{\mathrm{BFMC}}$ \\
\hline $\mathrm{H}^{\mathrm{a}}$ & $-0.42441(3)$ & $-0.50000(4)$ & & $0.540(2)$ & $0.700(2)$ \\
\hline $\mathrm{H}^{\mathrm{b}}$ & $-0.42440(2)$ & $-0.50000(2)$ & & $0.30223(1)$ & $0.31400(1)$ \\
\hline $\mathrm{H}_{2}^{+\mathrm{c}}$ & $-0.57683(2)$ & $-0.60263(2)$ & & $0.162207(8)$ & $0.2109(1)$ \\
\hline $\mathrm{H}_{2}^{+\mathrm{d}}$ & $-0.46772(6)$ & $-0.60264(5)$ & & $0.52249(2)$ & $0.53131(2)$ \\
\hline
\end{tabular}
Hartree).

${ }^{a}$ Gauß function $(\alpha=8 /(9 \pi))$, analytic: $E=-0.424413$ Hartree (expectation value), $\sigma=0.539609 .{ }^{b}$ Function with correct "cusp" behaviour $(\beta=1.848374)$, analytic: $E=-0.424413$ Hartree (expectation value), $\sigma=0.302233$. $^{\mathrm{c}}$ LCAO-formulation $(\alpha=$ $1.25), R_{\mathrm{AB}}=2.0$, analytic: $E=-0.576831$ Hartree (expectation value), $E_{\exp }[15],[16],[17] .{ }^{\mathrm{d}} e^{-\rho}$ with $F=1, r_{\mathrm{AB}}=2.0$, analytic: $E=-0.467741$ Hartree (expectation value).

Nevertheless the accuracy of the variance itself in the BFMC method is higher than in the LCAO case.

For two electron systems an explicit value for the parameter $V_{\max }$ has to be specified. We did an empirical optimisation for the He atom with our formulation as a guiding function. The value of $V_{\max }$ should roughly be constant for two electron systems because it depends mostly on the number of electron pairs. So we used the value $V_{\max }=15.0$ Hartree for all calculations of $\mathrm{He}$ and $\mathrm{H}_{2}$. In addition, for the hydrogen molecule an optimised single-zeta Hartree-Fock function was used. Again a difference between the one- and the two-centre case can be seen. For the He atom our formulation provides only half the standard deviation than the HF function. This causes significant improvements of the BFMC energy. For $\mathrm{H}_{2}$ the difference in the standard deviations of the two guiding functions is smaller than in the case of the hydrogen molecular ion and shows that the unphysical behaviour of our formulation between the nuclei is getting less important. An interesting point to remark is the different result for the BFMC energies of one system (e.g. He) calculated with different guiding functions. The solution for this surprising fact is that the results are influenced in a different way, depending on the guiding function used, from the approximation of the bounded potential.

For the $\mathrm{Be}$ atom we have to keep in mind that the method is now only exact within the fixed-node approximation which causes the largest error of course. Due to the limitations of the fixed-node approach, different guiding functions with the same node structure should produce the same BFMC result. As Table 3 shows, the results of the energies differ in a significant way in spite of the same node structure. The reason for that can be found in numerical problems 
Table 2. Results of the two electron systems (energies in Hartree).

\begin{tabular}{llllrrll}
\hline & $\langle E\rangle_{\mathrm{VMC}}$ & $\langle E\rangle_{\mathrm{BFMC}}$ & $E_{\mathrm{HF}-\text { limit }}$ & $E_{\exp }$ & $\%_{\text {corr }}$ & $\sigma_{\mathrm{VMC}}$ & $\sigma_{\mathrm{BFMC}}$ \\
\hline $\mathrm{He}^{\mathrm{a}}$ & $-2.84758(5)$ & $-2.90356(5)$ & -2.86168 & -2.903724 & 99.61 & $0.947(2)$ & $0.903(1)$ \\
$\mathrm{He}^{\mathrm{b}}$ & $-2.85549(4)$ & $-2.90376(3)$ & & & 100.09 & $0.4901(2)$ & $0.50452(1)$ \\
$\mathrm{H}_{2}^{\mathrm{c}}$ & $-1.12808(1)$ & $-1.17446(3)$ & -1.1336 & -1.1746 & 99.66 & $0.493(2)$ & $0.4238(8)$ \\
$\mathrm{H}_{2}^{\mathrm{d}}$ & $-1.03642(5)$ & $-1.1745(1)$ & & 99.76 & $0.57017(3)$ & $0.58384(5)$ \\
\hline
\end{tabular}

${ }^{\text {a }}$ Hartree-Fock single-zeta $(\zeta=11 / 16)$, analytic: $E=-2.847656$ Hartree (expectation value), $E_{\exp }[18] .{ }^{\mathrm{b}} e^{-\rho}$ with $F=1$, analytic: $E=-2.85551$ Hartree (expectation value). ${ }^{\mathrm{c}}$ Hartree-Fock single-zeta $(\zeta=1.2), r_{\mathrm{AB}}=1.4$, analytic: $E=-1.128077 \mathrm{Hartree}$ (expectation value), $E_{\exp }[18] .{ }^{\mathrm{d}} e^{-\rho}$ with $F=1, r_{\mathrm{AB}}=1.4$.

Table 3. Results for Be (energies in Hartree).

\begin{tabular}{llllllll}
\hline & $\langle E\rangle_{\mathrm{VMC}}$ & $\langle E\rangle_{\mathrm{BFMC}}$ & $E_{\mathrm{HF}-\text { limit }}$ & $E_{\exp }$ & $\%_{\text {corr }}$ & $\sigma_{\mathrm{VMC}}$ & $\sigma_{\mathrm{BFMC}}$ \\
\hline $\mathrm{Be}^{\mathrm{a}}$ & $-14.5567(2)$ & $-14.6494(5)$ & -14.5730 & -14.6674 & 80.93 & $2.130(3)$ & $2.090(2)$ \\
$\mathrm{Be}^{\mathrm{b}}$ & $-14.1549(2)$ & $-14.667(6)$ & & & 99.58 & $1.6194(3)$ & $1.987(7)$ \\
\hline
\end{tabular}

${ }^{\mathrm{a}}$ Hartree-Fock, single-zeta $\left(\zeta_{1 s}=3.68478, \zeta_{2 s}=0.95603\right)$, analytic: $E=-14.556740$ Hartree (expectation value). ${ }^{\mathrm{b}} e^{-\rho} F, \mathrm{~s}-\mathrm{Orbital}$ formulation, $E_{\exp }[21]$, [22].

which cause difficulties in the convergence behaviour and have been reported by other authors [19], [20] as well. Again the standard deviation of our formulation is lower than the one of a single-zeta $\mathrm{HF}$ function. Because of the six electron pairs a value of 90.0 Hartree was chosen for $V_{\max }$ (six times the value of the two electron systems). Notice that the results for the standard deviation itself gained by guiding functions with correct cusp-behaviour are one or two orders of magnitude more accurate than in the corresponding examples with guiding functions without this property.

\section{Comparison to Literature}

Because of the differences of the many Monte Carlo methods, the different computational efforts, and the varying numerical details in the different implementations, the comparison of our results with the literature is not an easy task. Anderson [23] gives a DMC energy of $E=-0.499995$ (4) Hartree for the hydrogen atom for a guiding function with an expectation value of $\langle E\rangle=-0.4998$ Hartree. In the same paper the DMC energy of the helium atom with the "single-zeta" Hartree-Fock function we used is given by $E=-2.9044 \pm 0.0012$ Hartree, and with a Hylleraas function with an expectation value of $\langle E\rangle=-2.902412$ Hartree a value of $E=-2.903649 \pm 0.00006$ Hartree is reported. For the hydrogen molecular ion a ground state energy of $E=-1.1745 \pm 0.0008$ Hartree can be found at Reynolds und Ceperley, who used a "single-zeta"-formulation with Jastrow factor $(\langle E\rangle=$ $-1.1507 \pm 0.0009$ Hartree). In the same paper a second function with clearly better expectation value gives, due to the higher computational effort, only a result with larger error bar. The ground state of the $\mathrm{Be}$ atom is given by Umrigar, Nightingale, and Runge [19] for a one determinant function with Jastrow factor $(\langle E\rangle=-14.6275(1)$ Hartree) by $E=$ -14.6569 (2) Hartree. The use of a multi-determinant function $(\langle E\rangle=-14.66648$ (1) Hartree) gives an energy of $E=-14.66717$ (3) Hartree. In an article of Moskowitz, Schmidt et al. [21] results for "guiding functions" with increasing complexity are given by $E_{1}=-14.640(6)$ Hartree, $E_{2}=-14.652$ (3) Hartree, $E_{3}=-14.665(2)$ Hartree and $E_{4}=-14.667(2)$ Hartree (all calculations within the fixed-node approximation).

\section{Conclusion}

The use of the $e^{-\rho}$-formulation with $F=1$ as a guiding function in the BFMC for systems up to two electrons turns out to be a good choice. It provides a very simple expression for the function itself and its corresponding local energy, whose boundaries can be analytically determined. The implementation of a computer program is of a very uncomplicated and general form. Beyond that, no parameters have to be determined or optimised in foregoing costly calculations. In spite of the extraordinary simple concept, the comparison with the literature shows that our results 
are of an equal or sometimes even better accuracy. From this point of view the increasing complexity of commonly used guiding functions has to be seen critically, especially if the computational effort for elaborating the guiding function itself by determining their parameters is taken into account. Today, where cheap and powerful computers are available the use of quick and dirty algorithms with guiding functions of a minimum complexity should be an interesting alternative for the computational practice. For systems with more than two electrons the quality of the results is mainly determined by the node structures of the guiding functions when the common fixed-node approximation is applied. For these systems the use of a parameter-free guiding function is very difficult,

[1] QCPE Program No 657, Quantum Magic, B. L. Hammond.

[2] F. F. Seelig, Z. Naturforsch. 21 a, 1500 (1966).

[3] F. F. Seelig, Int. J. Quant. Chem. 1, 809 (1967).

[4] G. Becker, Dissertation (Eberhard-Karls-Universität Tübingen 1996).

[5] T. Kato, Commun. Pure Appl. Math. 10, 151 (1957).

[6] M. H. Kalos, Phys. Rev. 128, 1791 (1962).

[7] J. B. Anderson, Understanding Chemical Reactivity: Quantum Mechanical Electronic Structure Calculations with Chemical Accuracy, edited by S. R. Langhoff, Kluwer Academic Publishers, New York 1995.

[8] J. B. Anderson, J. Chem. Phys. 65, 4121 (1976).

[9] J. B. Anderson, J. Chem. Phys. 86, 2839 (1987).

[10] D. M. Ceperley and B. J. Alder, J. Chem. Phys. 81, 5833 (1984).

[11] B. L. Hammond, W. A. Lester, and P. J. Reynolds, World Scientific Lecture and Course Notes in Chemistry, Vol. 1, Monte Carlo Methods in Ab Initio Quantum Chemistry, World Scientific, Singapore 1994. so that there seems to be no alternative to the usual method to use an optimised HF-determinant as part of the function. Another important point to keep in mind is the existence of numerical effects of a chaotic type, which sometimes cause convergence problems and have to be examined further in detail.

\section{Acknowledgement}

We wish to thank the "Studienstiftung des Deutschen Volkes" for giving a grant to one of us (C. M). We also wish to thank Dr. G. Becker and Dr. D. Hoffmann for many useful discussions and their kind support.

[12] M. Abramowitz and I. Stegun, Handbook of Mathematical Functions, Dover, New York 1972, p. 444.

[13] D. M. Ceperley, J. Stat. Phys. 65, 1237 (1991).

[14] W. A. Glauser, W. R. Brown, W. A. Lester jr. , D. Bressanini, B. L. Hammond, and M. L. Koszykowski, J. Chem. Phys. 97, 9200 (1992).

[15] D. R. Bates, K. Ledsham, and A. L. Stewart, Phil. Trans. A 246, 215 (1953).

[16] D. R. Bates and T. R. Carlson, Proc. Roy. Soc. (London) A 234, 207 (1956).

[17] L. Y. Wilson and G. A. Gallup, J. Chem. Phys. 45, 586 (1966).

[18] C. L. Pekeris, Phys. Rev. 51, 1649 (1958).

[19] C. J. Umrigar, M. P. Nightingale, and K. J. Runge, J. Chem. Phys. 99, 2865 (1993).

[20] J. Schütt and M. C. Böhm, in press.

[21] J. W. Moskowitz, K. E. Schmidt, M. A. Lee, and M. H. Kalos, J. Chem. Phys. 76, 1064 (1982).

[22] L. Szasz and J. Byrne, Phys. Rev. 158, 34 (1967).

[23] J. B. Anderson, J. Chem. Phys. 73, 3897 (1980). 